\title{
Relationship Between the Long-Term Preventive Effect of Combined Treatment With Antiarrhythmic Drugs Plus Angiotensin-Converting Enzyme Inhibitors and Circadian Variation in the Onset of Paroxysmal Atrial Fibrillation
}

\author{
Takashi Komatsu, ${ }^{1}$ MD, Hideaki Tachibana, ${ }^{1}$ MD, Yoshihiro Satoh, ${ }^{1} \mathrm{MD}$, Mahito Ozawa, ${ }^{1} \mathrm{MD}$, \\ Fusanori Kunugida, ${ }^{1} \mathrm{MD}$, and Motoyuki NaKamura, ${ }^{1}$ MD
}

\section{SUMMARY}

We examined the relationship between the efficacy of combined treatment with antiarrhythmic drugs (AAD) plus enalapril for maintaining sinus rhythm and circadian variation in the onset of paroxysmal AF.

Three hundred and forty-four patients with paroxysmal AF (239 men, mean age, $69 \pm 11$ years) who could be followed up $\geq 12$ months were divided into 3 groups on the basis of circadian variation in the onset of AF: a diurnal group (7:00 AM-5:00 PM, $n=57$ ), a nocturnal group (5:00 PM-7:00 AM, $n=108$ ), and a mixed group (onset during both periods, $n=169$ ). The maintenance rate of sinus rhythm during the follow-up period was compared between combined therapy (AAD plus enalapril) and AAD alone.

In the diurnal group, the maintenance rates of sinus rhythm at 12, 36, 60, and 90 months were $100 \%, 100 \%, 100 \%$, and $100 \%$, respectively, for patients treated with AAD plus enalapril $(n=22)$ versus $97 \%, 91 \%, 89 \%$, and $80 \%$ for patients treated with AAD alone $(n=35, P<0.05)$. In the nocturnal group, the maintenance rates of sinus rhythm at 12 , 36,60 , and 90 months were $96 \%, 96 \%, 96 \%$, and $92 \%$, respectively, in patients treated with AAD plus enalapril $(n=24)$ versus $100 \%, 100 \%, 100 \%$, and $100 \%$ in patients treated with AAD alone $(n=84, P=\mathrm{NS})$. In the mixed group, maintenance rates of sinus rhythm at $12,36,60$, and 90 months were $90 \%, 71 \%, 61 \%$, and $57 \%$, respectively, in patients treated with AAD plus enalapril $(n=49)$ versus $88 \%, 78 \%, 68 \%$, and $61 \%$ in patients treated with AAD alone $(n=120$, $P=\mathrm{NS})$.

Our findings suggest that the preventive efficacy of combined therapy with AAD plus enalapril is dependent on the timing of onset of paroxysmal $\mathrm{AF}$, and this regimen seems to be most beneficial for the diurnal type of paroxysmal $\mathrm{AF}$. (Int Heart J 2010; 51: 331-336)

Key words: Paroxysmal atrial fibrillation, Antiarrhythmic drug, Prevention, Angiotensin-converting enzyme inhibitor, Upstream therapy

$\mathrm{L}$ arge-scale long-term epidemiological studies conducted in Europe and the United States have shown that $\mathrm{AF}$ is an independent risk factor for cardiovascular death. ${ }^{1)}$ The number of patients with AF in the United States exceeded 5 million in 2000 and is expected to increase two to threefold over the next 50 years. ${ }^{2}$ In Japan, the population is aging rapidly and the prevalence of AF among elderly persons aged 70 years or older is already around 3\%. This is expected to increase to about $4.5 \%$ over the next 20 years. ${ }^{3)}$ Antiarrhythmic drug (AAD) therapy for the maintenance of sinus rhythm in patients with $\mathrm{AF}$ has limitations. ${ }^{4}$ Accordingly, attention has recently been paid to upstream treatment, in which AAD therapy is combined with renin-angiotensin-aldosterone system (RAAS) inhibitors ${ }^{5,6)}$ or statins ${ }^{7)}$ to modify the arrhythmic substrate of the atrial myocardium which is the underlying cause of AF. In a recent large clinical trial, however, additional treatment with angiotensin II-receptor blockers (ARB) to AAD was not associated with a reduction in the incidence of recurrent atrial fibrillation. ${ }^{8)}$ It is still not clear which clinical profile of paroxysmal AF benefits from the addition of RAAS inhibitor therapy as an upstream treatment.

We report on the results of our investigation into the longterm preventive effect of combined treatment with enalapril, an angiotensin-converting enzyme inhibitor (ACEI), plus AAD therapy in relation to circadian variation in the onset of paroxysmal AF.

\section{MeTHODS}

Subjects: The subjects were 344 patients (239 men and 105 women, average age, $69 \pm 11$ years) who had symptoms such as palpitations and electrocardiographic evidence of AF corresponding to the symptoms. All patients attended the hospital

From the ${ }^{1}$ Division of Cardiology, Department of Internal Medicine and Memorial Heart Center, Iwate Medical University School of Medicine, Iwate, Japan.

Address for correspondence: Takashi Komatsu, MD, Division of Cardiology, Department of Internal Medicine and Memorial Heart Center, Iwate Medical University, 19-1 Uchimaru, Morioka, Iwate 020-8505, Japan.

Received for publication April 5, 2010.

Revised and accepted June 28, 2010. 
every 2 to 4 weeks and could be followed-up for more than one year. To detect underlying cardiopulmonary disease, all subjects were interviewed to obtain a medical history and underwent noninvasive tests such as chest X-rays, exercise stress testing, and transthoracic echocardiography. If deemed necessary by their attending physician, they also underwent pulmonary function tests, chest computed tomography, and cardiac catheterization. Patients were excluded if they had serious bradyarrhythmia (sick sinus syndrome, atrioventricular block, or intraventricular conduction defect), hepatic or renal dysfunction, pregnancy or the possibility of pregnancy, or if they were taking beta-blockers.

Patients were divided into 3 groups according to time of AF occurrence, which was determined from the results of electrocardiography (ECG) and ambulatory 24-hour ECG recording, or if AF had already been diagnosed at the time of consultation, from the times when the same symptoms appeared. The 3 groups consisted of a diurnal group (onset 7:00 AM - 5:00 $\mathrm{PM}, n=57$ ), nocturnal group (onset 5:00 - 7:00 AM, $n=108$ ), and mixed group (onset during both periods, $n=169$ ). ${ }^{4)}$ The efficacy for prevention of permanent AF was compared between patients treated with both AAD and enalapril and those treated with $\mathrm{AAD}$ alone in each of the 3 groups. This investigation was conducted from June 1997 to August 2009, and the mean follow-up period was $60 \pm 35$ months.

Defibrillation protocol and AAD therapy: When AF had persisted for less than 48 hours, defibrillation was attempted immediately by drug therapy or by electrical defibrillation under intravenous anesthesia with thiopental according to the American Heart Association (AHA) guidelines. ${ }^{9)}$ If AF had persisted for more than 48 hours in patients who were not on anticoagulant therapy, transesophageal echocardiography was performed to confirm that there was no left atrial thrombosis or spontaneous echo contrast, after which defibrillation was performed and anticoagulant therapy with warfarin was added. If the patients were already receiving anticoagulant therapy, electrical defibrillation was performed after their PT-INR was confirmed to be between 1.6 and 3.0.

According to the protocol for preventing the recurrence of $\mathrm{AF}$ by $\mathrm{AAD}$, patients with a left ventricular ejection fraction of more than $40 \%$ on echocardiography underwent medical or electrical defibrillation to restore sinus rhythm, and were subsequently randomized by the envelope method to either class Ia or Ib AAD (disopyramide $300 \mathrm{mg}$ /day, aprindine $60 \mathrm{mg}$ /day, or cibenzoline $300 \mathrm{mg} /$ day) as first-line therapy. Patients were carefully observed for recurrence of $\mathrm{AF}$, and if this was detected during follow-up, defibrillation was repeated at that time. Patients were subsequently randomized to either class Ic AAD (flecainide $150 \mathrm{mg} /$ day or pilsicainide $150 \mathrm{mg}$ /day) or to receive bepridil (150 mg/day) as second-line therapy and were observed carefully for recurrence. If AF recurred in a patient on the second-line therapy, amiodarone was administered if the patient agreed. If consent was not given, they received a drug from Class I AAD that had not been administered already as third-line therapy. Patients whose left ventricular ejection fraction was less than $40 \%$ on transthoracic echocardiography underwent electrical defibrillation to restore sinus rhythm, and were subsequently treated with either aprindine $(60 \mathrm{mg} /$ day $)$ or bepridil ( $150 \mathrm{mg} /$ day) as first- or second-line therapy. If AF recurred despite such therapy, amiodarone was administered as the third-line drug at a starting dose of $400 \mathrm{mg} /$ day for two weeks and then at $200 \mathrm{mg} /$ day thereafter. ${ }^{4,6)}$ Enalapril (5 mg/ day) was only administered to paroxysmal AF patients with hypertension or cardiac dysfunction.

Standard 12-lead ECG and ambulatory 24-hour ECG recordings were performed after commencement of drug therapy and at 2 to 4 weeks after drugs were changed. At outpatient consultations, ambulatory electrocardiographic monitoring with an IEC-1101 "Heart Mate" (Nihon Koden Corporation, Tokyo) was performed to confirm that sinus rhythm was being maintained. In addition, if patients experienced symptoms such as palpitations and precordial discomfort, ambulatory 24-hour ECG recordings were performed to confirm the recurrence of $\mathrm{AF}$ if the physician considered this to be required.

Definitions: Paroxysmal AF was defined as AF that was confirmed to cease within a week based on symptoms and the results of ambulatory 24-hour ECG ${ }^{10)}$ while permanent AF was defined as AF that showed a refractory course despite treatment with AAD, so that sinus rhythm was not restored for at least 6 months. Recurrence of AF was defined as the time when AF was first confirmed by electrocardiography after commencement of AAD. Hypertension was defined as a casual systolic blood pressure $\geq 140 \mathrm{mmHg}$ or diastolic blood pressure $\geq 90 \mathrm{mmHg},{ }^{11)}$ and cerebral thromboembolism was diagnosed from the presence of relevant symptoms and detection of an infarct more than $3 \mathrm{~mm}$ in size on brain computed tomographic examination or magnetic resonance imaging, which was performed in all patients.

Statistical analysis: Demographic data on patients and frequency of recurrence of AF are expressed as the mean \pm standard deviation. Clinical characteristics and AF recurrence in individual patients were compared between the two groups by unpaired $t$-test for continuous variables and by the chi-square test for categorical ones. Survival curves were estimated by the Kaplan-Meier method and were compared by the log-rank test (Cox-Mantel). A $P<0.05$ was considered as statistically significant.

Table I. Clinical Characteristics of Patients With Diurnal AF

\begin{tabular}{lccc}
\hline & $\begin{array}{c}\text { Enalapril (+) } \\
\text { group }\end{array}$ & $\begin{array}{c}\text { Enalapril (-) } \\
\text { group }\end{array}$ & $P$ \\
\hline Number & 22 & 35 & \\
Age (years) & $69 \pm 8$ & $72 \pm 10$ & $\mathrm{NS}$ \\
Gender (male:female) & $13: 9$ & $24: 11$ & $\mathrm{NS}$ \\
Smoking & $8(36 \%)$ & $10(29 \%)$ & $\mathrm{NS}$ \\
Hypertension & $19(86 \%)$ & $15(51 \%)$ & $P<0.05$ \\
Diabetes mellitus & $3(14 \%)$ & $5(14 \%)$ & $\mathrm{NS}$ \\
Hyperlipidemia & $4(18 \%)$ & $5(14 \%)$ & $\mathrm{NS}$ \\
Hyperuricemia & $1(5 \%)$ & $2(6 \%)$ & $\mathrm{NS}$ \\
Alcohol habit & $8(36 \%)$ & $13(37 \%)$ & $\mathrm{NS}$ \\
Organic heart disease & $10(45 \%)$ & $13(37 \%)$ & $\mathrm{NS}$ \\
Organic pulmonary disease & $3(14 \%)$ & $1(3 \%)$ & $\mathrm{NS}$ \\
Thromboembolism & $2(9 \%)$ & $6(17 \%)$ & $\mathrm{NS}$ \\
AF history(months) & $8 \pm 16$ & $9 \pm 19$ & $\mathrm{NS}$ \\
TTE parameters; & & & \\
LVDd (mm) & $46.7 \pm 5.7$ & $44.5 \pm 5.2$ & $\mathrm{NS}$ \\
Left atrium dimension (mm) & $34.0 \pm 7.6$ & $35.3 \pm 6.4$ & $\mathrm{NS}$ \\
Ejection fraction $(\%)$ & $67.8 \pm 12.0$ & $70.8 \pm 11.7$ & $\mathrm{NS}$ \\
ANP during sinus rhythm & $32.2 \pm 20.0$ & $35.8 \pm 21.8$ & $\mathrm{NS}$ \\
$\quad$ (pg/mL) & & & \\
\hline LV & & & \\
\hline
\end{tabular}

LVDd indicates left ventricular diastolic dimension; TTE, transthoracic echocardiography; ANP, atrial natriuretic peptide; and AF, atrial fibrillation. 


\section{RESULTS}

Diurnal AF group: The clinical characteristics of patients with diurnal AF are shown in Table I. Although hypertension was significantly $(P<0.05)$ more common in patients treated with AAD and enalapril $(n=22)$ than in patients treated with AAD alone $(n=35)$, no significant differences in other background factors were observed. Survival rates free from conversion to permanent AF were $100 \%$ at 12, 24, 36, 60, and 90 months for patients treated with $\mathrm{AAD}$ and enalapril, while the respective rates were $97 \%, 94 \%, 91 \%, 89 \%$, and $80 \%$ for patients treated with AAD alone. These rates were significantly lower at 90 months in patients treated with AAD alone than in patients treated with AAD and enalapril $(P<0.05$, Figure 1$)$.

On the other hand, survival rates free from conversion to permanent $\mathrm{AF}$ during the follow-up period were $86 \%$ for pa-

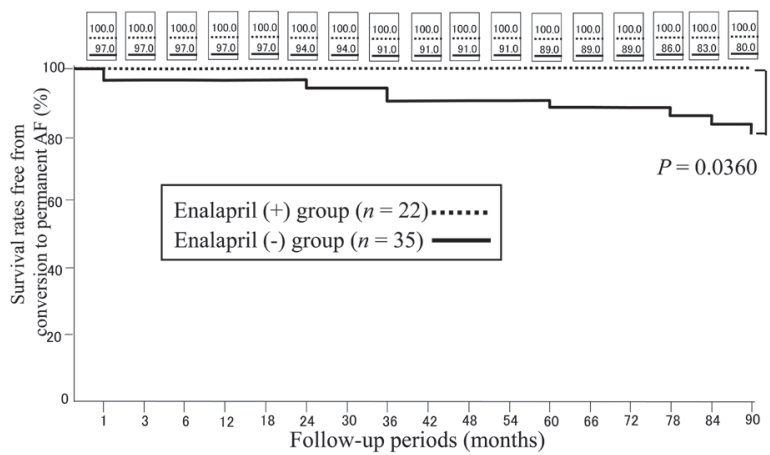

Figure 1. Actuarial rates for prevention of permanent paroxysmal atrial fibrillation in patients with diurnal type PAF.

Table II. Clinical Characteristics of Patients With Nocturnal AF

\begin{tabular}{lccc}
\hline & $\begin{array}{c}\text { Enalapril (+) } \\
\text { group }\end{array}$ & $\begin{array}{c}\text { Enalapril (-) } \\
\text { group }\end{array}$ & $P$ \\
\hline Number & 24 & 84 & \\
Age (years) & $70 \pm 9$ & $61 \pm 14$ & $P<0.05$ \\
Gender (male:female) & $15: 9$ & $64: 20$ & NS \\
Smoking & $6(25 \%)$ & $28(33 \%)$ & NS \\
Hypertension & $17(71 \%)$ & $22(26 \%)$ & $P<0.01$ \\
Diabetes mellitus & $4(17 \%)$ & $6(7 \%)$ & NS \\
Hyperlipidemia & $2(8 \%)$ & $13(15 \%)$ & NS \\
Hyperuricemia & $2(8 \%)$ & $7(8 \%)$ & NS \\
Alcohol habit & $9(38 \%)$ & $45(54 \%)$ & NS \\
Organic heart disease & $10(42 \%)$ & $17(20 \%)$ & NS \\
Organic pulmonary disease & $3(13 \%)$ & $7(8 \%)$ & NS \\
Thromboembolism & $5(21 \%)$ & $9(11 \%)$ & NS \\
AF history (months) & $17 \pm 52$ & $17 \pm 24$ & NS \\
$\quad$ TTE parameters; & & & \\
LVDD (mm) & $47.6 \pm 6.3$ & $45.9 \pm 5.3$ & NS \\
Left atrium dimension (mm) & $34.8 \pm 5.4$ & $32.9 \pm 5.6$ & NS \\
Ejection fraction (\%) & $67.5 \pm 11.2$ & $70.6 \pm 8.3$ & NS \\
ANP during sinus rhythm & $57.0 \pm 45.3$ & $37.3 \pm 24.4$ & NS \\
$\quad$ (pg/mL) & & & \\
\hline
\end{tabular}

LVDd indicates left ventricular diastolic dimension; TTE, transthoracic echocardiography; ANP, atrial natriuretic peptide; and AF, atrial fibrillation. tients treated with bepridil or amiodarone therapy $(n=17)$, and $90 \%$ for those treated without bepridil or amiodarone therapy $(n=40)$. There was no significant difference in the rate between two groups.

Nocturnal AF group: The clinical characteristics of patients with nocturnal AF are shown in Table II. Although age and prevalence of hypertension were significantly higher $(P<0.05)$ in patients treated with AAD and enalapril $(n=24)$ than in patients treated with AAD alone $(n=84)$, no significant differences were observed for other background factors. The survival rates free from conversion to permanent $\mathrm{AF}$ were $96 \%, 96 \%$, $96 \%, 96 \%$, and $92 \%$ at $12,24,36,60$, and 90 months, respectively, for the patients treated with AAD and enalapril, while the corresponding rates were $100 \%$ at all of these times for the patients treated with AAD alone. No significant difference was observed between the two groups at 90 months $(P=$ NS, Figure 2).

Mixed AF group: The clinical characteristics of patients with

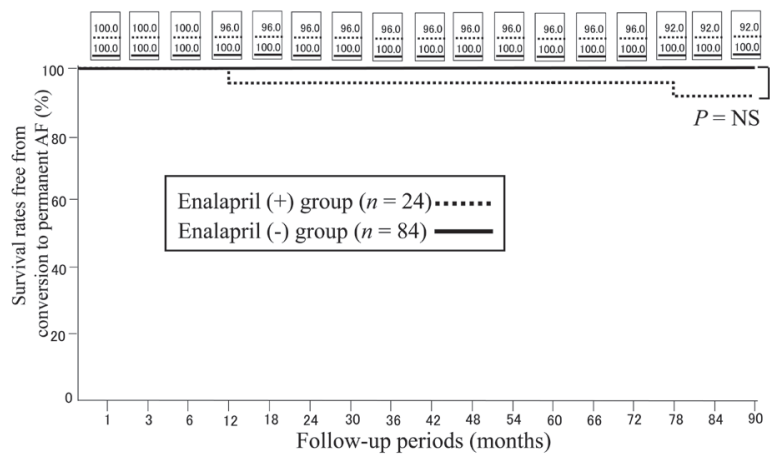

Figure 2. Actuarial rates for prevention of permanent paroxysmal atrial fibrillation in patients with nocturnal type PAF.

Table III. Clinical Characteristics of Patients With Mixed AF

\begin{tabular}{lccc}
\hline & $\begin{array}{c}\text { Enalapril (+) } \\
\text { group }\end{array}$ & $\begin{array}{c}\text { Enalapril (-) } \\
\text { group }\end{array}$ & $P$ \\
\hline Number & 49 & 120 & \\
Age (years) & $71 \pm 8$ & $67 \pm 11$ & NS \\
Gender (male:female) & $29: 20$ & $84: 36$ & NS \\
Smoking & $14(29 \%)$ & $36(30 \%)$ & NS \\
Hypertension & $28(57 \%)$ & $44(37 \%)$ & $P<0.05$ \\
Diabetes mellitus & $12(24 \%)$ & $13(11 \%)$ & $P<0.05$ \\
Hyperlipidemia & $3(6 \%)$ & $13(11 \%)$ & NS \\
Hyperuricemia & $4(8 \%)$ & $5(4 \%)$ & NS \\
Alcohol habit & $19(39 \%)$ & $60(50 \%)$ & NS \\
Organic heart disease & $22(45 \%)$ & $39(33 \%)$ & NS \\
Organic pulmonary disease & $5(10 \%)$ & $8(7 \%)$ & NS \\
Thromboembolism & $18(37 \%)$ & $27(23 \%)$ & NS \\
AF history (months) & $16 \pm 26$ & $23 \pm 32$ & NS \\
$\quad$ TTE parameters; & & & \\
LVDD (mm) & $48.2 \pm 5.2$ & $45.5 \pm 5.9$ & NS \\
Left atrium dimension (mm) & $37.7 \pm 6.2$ & $34.6 \pm 6.7$ & NS \\
Ejection fraction (\%) & $60.9 \pm 13.8$ & $68.6 \pm 10.5$ & NS \\
ANP during sinus rhythm & $63.0 \pm 64.4$ & $45.8 \pm 40.9$ & NS \\
$\quad$ (pg/mL) & & & \\
\hline
\end{tabular}

LVDd indicates left ventricular diastolic dimension; TTE, transthoracic echocardiography; ANP, atrial natriuretic peptide; and AF, atrial fibrillation. 


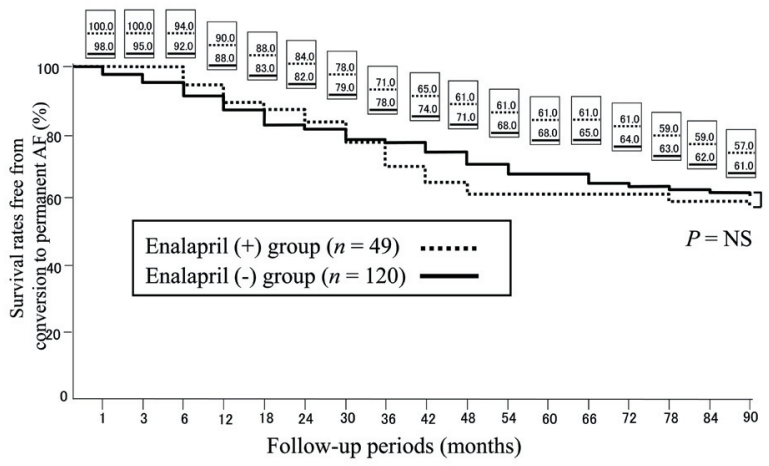

Figure 3. Actuarial rates for prevention of permanent paroxysmal atrial fibrillation in patients with mixed type PAF.

mixed AF are shown in Table III. Although the prevalence of hypertension and diabetes mellitus was significantly higher $(P$ $<0.05)$ in patients treated with AAD and enalapril than in patients treated with AAD alone $(n=120)$, no significant differences were observed for other background factors. The survival rate free from conversion to permanent $\mathrm{AF}$ was $90 \%, 84 \%$, $71 \%, 61 \%$, and $57 \%$ at $12,24,36,60$, and 90 months, respectively, for patients treated with AAD and enalapril $(n=49)$, and $88 \%, 82 \%, 78 \%, 68 \%$, and $61 \%$ for patients treated with AAD alone. There was no significant difference between the two groups at 90 months ( $P=$ NS, Figure 3$)$.

Adverse events requiring discontinuation of enalapril: Of the 99 patients who received enalapril during the follow-up period, administration was discontinued in 4 patients $(4.2 \%)$ due to dry cough. No other adverse or fatal drug reactions were observed.

\section{DisCUSSION}

Main finding: This study showed that upstream treatment of AF with a combination of AAD and enalapril had a beneficial effect in preventing AF from becoming permanent in patients with diurnal AF. The findings also suggested that combined therapy with enalapril had a differential efficacy for maintaining sinus rhythm depending on the type of onset of paroxysmal AF. As far as we could determine, there have been no previous reports describing the long-term effect of combined therapy with enalapril in relation to circadian variation in AF occurrence.

Influence of autonomic nervous system on paroxysmal AF: Yamashita, et al reported an analysis of 407 arrhythmic events in 150 patients who had paroxysmal AF while not on medication and they concluded that paroxysmal AF showed circadian variation. It occurred most frequently in the daytime with a single peak in elderly patients over 60 years old, while younger patients under 60 years of age showed a different pattern of circadian variation with 3 peaks after meals until midnight. ${ }^{12)}$ That is, they found that the relationship between paroxysmal AF and activity of the autonomic nervous system varies with age, and that sympathetic activity in elderly people and parasympathetic (vagal) activity in younger people are intimately related to the onset of paroxysmal AF. In the past, we have similarly reported that the preventive effect of AAD varies among patients with paroxysmal AF because the pharmacological blocking of channels is up-regulated by vagotonia as well as sympathicotonia. ${ }^{13)}$ Likewise, several studies have categorized paroxysmal AF into sympathetic-dependent and parasympathetic-dependent types based on analysis of heart rate changes before the onset of arrhythmia by ambulatory 24-hour ECG ${ }^{14,15)}$ and on the clinical characteristics of paroxysmal $\mathrm{AF}^{16)}$

There also have been reports that the atrial refractory period was shortened by either sympathetic or parasympathetic stimulation in an experimental model of paroxysmal AF, and that the heterogeneity of atrial refractoriness was increased by parasympathetic stimulation, making it easier to induce and maintain $\mathrm{AF}^{17,18)}$

Preventive effect of RAAS inhibitors: It has been reported that persistent tachycardia increases blood levels of angiotensin II in an experimental model of rapid pacing, ${ }^{19)}$ while both angiotensin-converting enzyme and intracellular regulatory kinase Erk1/Erk2 show increased activity in patients with $\mathrm{AF}^{20)}$ The elevation of left atrial pressure associated with increased angiotensin II causes intracellular calcium overload by activating the stretch-activated channel ${ }^{21)}$ and also through another mechanism via G-proteins connected to AT1 receptors, leading to a shorter action potential duration. As a result, the effective refractory period of the atrial myocardium is shortened and the heterogeneity of refractoriness is increased, so that supraventricular extrasystoles that can trigger AF are more likely to occur due to triggered activity and increased abnormal automaticity. ${ }^{22,23)}$ Moreover, increased ventricular wall stress promotes cardiomyocyte degeneration and fibrosis by activation of the Erk cascade via the angiotensin II receptor. ${ }^{20)}$ Based on the above findings, RAAS inhibitors are not only expected to improve cardiac hemodynamics and reduce atrial pressure and wall stress, but also to correct the neurohumoral abnormalities that promote cardiomyocyte fibrosis and degeneration. ${ }^{24,25)}$ Furthermore, RAAS inhibitors have recently been shown to suppress apoptosis ${ }^{26}$ and to have an anti-inflammatory effect, ${ }^{27)}$ which are possible additional mechanisms leading to a preventive effect on AF. There have already been some reports that combined therapy with RAAS inhibitors is effective for preventing paroxysmal and persistent $\mathrm{AF}$ from recurring and becoming permanent. ${ }^{6,28)}$

Effect of enalapril in patients with diurnal AF: Zankov, et al reported that RAAS inhibitors suppress the activation of delayed rectifier Iks channels via angiotensin II. ${ }^{29)}$ Assuming that a shorter refractory period due to activation of Iks channels by sympathetic hypertonia is involved in the onset and maintenance of diurnal AF, it would be expected that additional treatment with an RAAS inhibitor, which suppresses Iks channels, would have a beneficial effect for preventing AF. In addition, activation of the L-type calcium current via sympathetic hypertonia not only shortens the action potential duration due to an increase in intracellular calcium, but also promotes supraventricular extrasystoles via increased automaticity or triggered activity. ${ }^{22,23)}$ Thus, RAAS inhibitors are expected to be especially effective for diurnal AF because these drugs suppress sympathetic activity via beta-receptor blockade. ${ }^{30)}$ The inhibitory effect of RAAS inhibitors on the sympathetic activity may also help to prevent abnormal autonomic remodeling caused by persistent $\mathrm{AF}^{31,32)}$

Limitations: The limitations of this study are as follows. First, 
it was a retrospective observational investigation and therefore there was bias in the patient background factors. This is due to the use of enalapril for the 3 groups of AF categorized by the timing of onset being partly determined by the presence of hypertension and cardiac dysfunction. Second, several antiarrhythmic agents were used for evaluation of combination therapy with enalapril, but a separate Japanese study has shown that the preventive effects of these drugs vary. ${ }^{4)}$ Third, there is a limit with respect to the accuracy of assessing the recurrence of AF based on either symptoms or electrocardiography. A study using ambulatory 24-hour ECG monitoring showed that even patients with symptomatic paroxysmal AF were not aware of the occurrence of tachycardia on more than half of all occasions. ${ }^{33)}$ On the other hand, a study that employed a portable electrocardiographic recording device (Cardiophone) showed that 30 to $70 \%$ of episodes of palpitations noted by patients with symptomatic AF were due to sinus tachycardia or atrial extrasystoles. ${ }^{34)}$ That is, at present there are methodological limitations to the accurate assessment of $\mathrm{AF}$ recurrence based on either patient symptoms or the results of electrocardiography. Finally, the total number of patients was relatively small. More patients are required to draw a definite conclusion in terms of the efficacy of combined treatment with enalapril plus AAD therapy in relation to circadian variation in the onset of paroxysmal AF.

Therefore, the preventive effect of RAAS inhibitors on the different types of paroxysmal AF in Japanese patients needs to be re-evaluated by prospective multicenter controlled studies in the future.

Conclusion: This study suggests that the preventive effect of combined treatment with AAD and enalapril depends on the type of AF occurrence, and is expected to be most beneficial for the diurnal type of paroxysmal AF.

\section{ACKNOWLEDGMENTS}

The authors would like to thank all study participants as well as the physicians and medical staff of Iwate Medical University School. We are also deeply grateful for the direction provided by Professor Ken Okumura of Hirosaki University School of Medicine (Aomori, Japan).

\section{REFERENCES}

1. Kannel WB, Abbott RD, Savage DD, McNamara PM. Epidemiologic features of chronic atrial fibrillation: the Framingham study. N Engl J Med 1982; 306: 1018-22.

2. Miyasaka Y, Barnes ME, Gersh SJ, et al. Secular trends in incidence of atrial fibrillation in Olmsted County, Minnesota, 1980 to 2000 , and implications on the projections for future prevalence. Circulation 2006; 114: 119-25.

3. Ohsawa M, Okayama A, Sakata K, et al. Rapid increase in estimated number of persons with atrial fibrillation in Japan: an analysis from national surveys on cardiovascular diseases in 1980, 1990 and 2000. J Epidemol 2005; 15: 194-6.

4. Komatsu T, Nakamura S, Suzuki O, Horiuchi D, Yomogida K Okumura K. Long-term prognosis of patients with paroxysmal atrial fibrillation depends on their response to antiarrhythmic therapy. Circ J 2004; 68: 729-33.

5. Healey JS, Baranchuk A, Crystal E, et al. Prevention of atrial fibrillation with angiotensin-converting enzyme inhibitors and angiotensin receptor blockers: a meta-analysis. J Am Coll Cardiol
2005; 45: 1832-9.

6. Komatsu T, Ozawa M, Tachibana $\mathrm{H}$, et al. Combination therapy with amiodarone and enalapril in patients with paroxysmal atrial fibrillation prevents the development of structural atrial remodeling. Int Heart J 2008; 49: 435-47.

7. Fauchier L, Pierre B, de Labriolle A, Grimard C, Zannad N, Babuty D. Antiarrhythmic effect of statin therapy and atrial fibrillation a meta-analysis of randomized controlled trials. J Am Coll Cardiol 2008; 51: 828-35.

8. GISSI-AF investigators, Disertori M, Latini R, et al. Valsartan for prevention of recurrent atrial fibrillation. N Engl J Med 2009; 360: 1606-17.

9. Fuster V, Rydén LE, Cannom DS, et al. ACC/AHA/ESC 2006 guidelines for the management of patients with atrial fibrillation: executive summary. A report of the American College of Cardiology/American Heart Association Task Force on Practice Guidelines and the European Society of Cardiology Committee for Practice Guidelines. Developed in Collaboration With the European Heart Rhythm Association and the Heart Rhythm Society. Circulation 2006; 114: 700-52.

10. Sopher SM, Camm AJ. Atrial fibrillation: maintenance of sinus rhythm versus rate control. Am J Cardiol 1996; 77: 24A-37A. (Review)

11. European Society of Hypertension-European Society of Cardiology Guidelines Committee. 2003 European Society of Hypertension-European Society of Cardiology guidelines for the management of arterial hypertension. J Hypertens 2003; 21: 1011-53.

12. Yamashita T, Murakawa Y, Hayami N, et al. Relation between aging and circadian variation of paroxysmal atrial fibrillation. Am J Cardiol 1998; 82: 1364-7.

13. Komatsu T, Satoh Y, Tachibana H, et al. Efficacy of long-term flecainide therapy in patients with paroxysmal atrial fibrillation. Analysis based on time of onset. J Arrhythmia 2006; 22: 37-43.

14. Yamaguchi I, Maeda H, Kurihara T, Sugishita Y. Baroreflex sensitivity of patients with paroxysmal atrial fibrillation. Jpn J Cardiac Pacing Electrophysiol 1991; 7: 360-4. (Japanese)

15. Saitoh K, Sasabe N, Osaka M, et al. Autonomic nervous activity preceding paroxysmal atrial fibrillation. Jpn J Electrophysiol 1993; 13: 181-8. (Japanese)

16. Coumel P. Autonomic arrhythmogenic factors in paroxysmal atrial fibrillation. Olsson SB, Allessie MA, Campbell RWF, editors. Atrial Fibrillation: Mechanisms and therapeutic strategies. Armonk: Futura Publishing Co; 1990. p.171-85.

17. Liu L, Nattel S. Differing sympathetic and vagal effects on atrial fibrillation in dogs: role of refractoriness heterogeneity. Am J Physiol 1997; 273: H805-16.

18. Tsujii H, Fujiki A, Tani M, Yoshida S, Sasayama S. Quantitative relationship between atrial refractoriness and the dispersion of refractoriness in atrial vulnerability. Pacing Clin Electrophysiol 1992; 15: 403-10.

19. Willems R, Sipido KR, Holemans P, Ector H, Van de Werf F, Heidbüchel H. Different patterns of angiotensin II and atrial natriuretic peptide secretion in a sheep model of atrial fibrillation. J Cardiovasc Electrophysiol 2001; 12: 1387-92.

20. Goette A, Staack T, Röcken C, et al. Increased expression of extracellular signal-regulated kinase angiotensin-converting enzyme in human atria during atrial fibrillation. J Am Coll Cardiol 2000; 35: 1669-77.

21. Bode F, Katchman A, Woosley RL, Franz MR. Gadolinium decreases stretch-induced vulnerability to atrial fibrillation. Circulation 2000; 101: 2200-5.

22. Goette A, Honeycutt C, Langberg JJ. Electrical remodeling in atrial fibrillation. Time course and mechanisms. Circulation 1996; 94 : 2968-74

23. Wijffels MC, Kirchhof CJ, Dorland R, Power J, Allessie MA. Electrical remodeling due to atrial fibrillation in chronically instrumented conscious goats: roles of neurohumoral changes, ischemia, atrial stretch, and high rate of electrical activation. Circulation 1997; 96: 3710-20.

24. Gottlieb SS, Diekstein K, Fleck E, et al. Hemodynamic and neu- 
rohormonal effects of the angiotensin II antagonist losartan in patients with congestive heart failure. Circulation 1993; 88: 1602-9.

25. Li D, Shi YF, Levi M, Benardeau A, Nattel S. Prevention of atrial structural remodeling: a potential new approach to treating atrial fibrillation. Circulation 1999; 100: I-11.

26. Cardin S, Li D, Thorin-Trescases N, Leung TK, Thorin E, Nattel S. Evolution of the atrial fibrillation substrate in experimental congestive heart failure: angiotensin-dependent and -independent pathways. Cardiovasc Res 2003; 60: 315-25.

27. Filser D, Buchholz K, Haller H, European Trial on Olmesartan and Pravastatin in Inflammation and Atherosclerosis (EUTOPIA) Investigators. Antiinflammatory effects of angiotensin II subtype 1 receptor blockade in hypertensive patients with microinflammation. Circulation 2004; 110: 1103-7.

28. Madrid AH, Bueno MG, Rebollo JM, et al. Use of irbesartan to maintain sinus rhythm in patients with long-lasting persistent atrial fibrillation: a prospective and randomized study. Circulation 2002; 106: 331-6.

29. Zankov DP, Omatsu-Kanbe M, Isono T, et al. Angiotensin II potentiates the slow component of delayed rectifier $\mathrm{K}+$ current via the AT1 receptor in guinea pig atrial myocytes. Circulation 2006; 113: $1278-86$

30. Lonn EM, Yusuf S, Jha P, et al. Emerging role of angiotensin-converting enzyme inhibitors in cardiac and vascular protection. Circulation 1994; 90: 2056-69. (Review)

31. Lombardi F, Colombo A, Basilico B, et al. Heart rate variability and early recurrence of atrial fibrillation after electrical cardioversion. J Am Coll Cardiol 2001; 37: 157-62.

32. Jayachandran JV, Sih HJ, Winkle W, Zipes DP, Hutchins GD, Olgin JE. Atrial fibrillation produced by prolonged rapid atrial pacing is associated with heterogeneous changes in atrial sympathetic innervation. Circulation 2000; 101: 1185-91.

33. Page RL, Wilkinson WE, Clair WK, McCarthy EA, Pritchett EL. Asymptomatic arrhythmias in patients with symptomatic paroxysmal atrial fibrillation and paroxysmal supraventricular tachycardia. Circulation 1994; 89: 224-7.

34. Fukuda Y, Akashi M, Noma S, Mitamura H, Ogawa S: Asymptomatic recurrence of atrial fibrillation - Cardiophone-guided management. Circ J 2003; 67(Suppl I):310. 